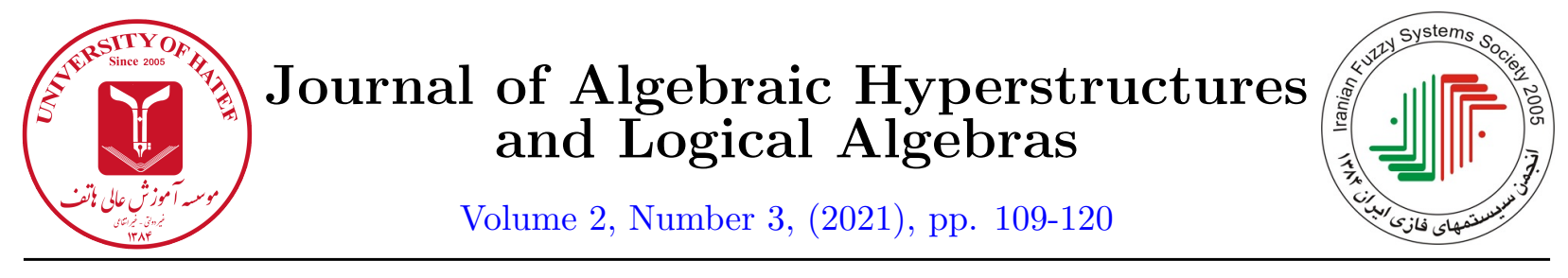

\title{
Minimal prime ideals in hoops
}

\author{
R.A. Borzooei ${ }^{1}$ and M. Aaly Kologani² \\ ${ }^{1}$ Department of Mathematics, Faculty of Mathematical Sciences, Shahid Beheshti University, Tehran, Iran \\ ${ }^{2}$ Hatef Higher Education Institute, Zahedan, Iran \\ borzooei@sbu.ac.ir, mona4011@gmail.com
}

"This paper is dedicated to Professor Young Bae Jun on the occasion of his 70th birthday."

\begin{abstract}
In this paper, we define the notion of minimal prime ideals of hoops and investigate some properties of them. Then by using the notion of annihilators, we study the relation between minimal prime ideals and annihilators. Also, we introduce the notion of zero divisors elements of hoops and prove that the set of all zero divisors of hoops is a union of all minimal prime ideals of hoop. Finally, by using the notions of minimal prime ideals and maximal ideals of hoop, we introduce two new ideals as $p$-ideal and $m$-ideal. Then we study some properties of them and investigate the relation between them and prove that every $p$-ideal of semi-simple hoop is an $m$-ideal of it.
\end{abstract}

\section{Article Information}

Corresponding Author:

M. Aaly Kologani;

Received: June 2021;

Accepted: August 2021;

Paper type: Original.

\section{Keywords:}

Hoop, ideal, prime ideal, minimal prime, annihilator, zero divisors.

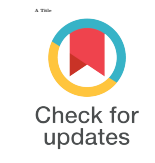

\section{Introduction}

It is well known that logic gives a technique for the articial intelligence to make the computers simulate human being in dealing with certainty and uncertainty in information. And as uncertain information processing, non-classical logic has become a formal and useful tool for computer science to deal with uncertain information, fuzzy information and intelligent system. Various logical algebras have been proposed and researched as the semantical systems of non-classical logical systems. Among these logical algebras, residuated lattices were introduced by Ward and Dilworth in 1939 to constitute the semantics of Höhle Monoidal Logic which are the basis for the majority of formal fuzzy logic. Apart from their logical interest, residuated lattices have interesting algebraic properties and include two important classes of algebras: BL-algebras and MV-algebras. In order to study the basic logic framework of fuzzy set system, based on continuous triangle module and

https://doi.org/10.52547/HATEF.JAHLA.2.3.9 
under the theoretical framework of residuated lattices theory, Hájek [9], proposed a new fuzzy logic system BL-system and the corresponding logical algebraic system BL-algebra. Hoops are naturally ordered commutative resituated integral monoids, which was introduced by Bosbach in [6, [7]. In recent decades, many mathematicians have worked on it and developed structure theory by using

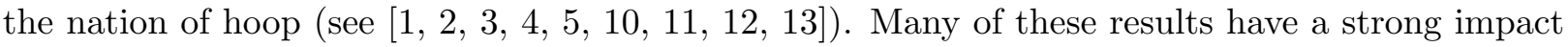
with fuzzy logic. Particularly, from the structure theorem of finite basic hoops one obtains an elegant short proof of the completeness theorem for propositional basic logic introduced by Hájek in [9]. The notion of ideals has been introduced in many algebraic structures such as lattices, rings, MV-algebras. Ideals theory is a very effective tool for studying various algebraic and logical systems. In the theory of MV-algebras the notion of ideals is at the center and deductive systems and ideals are dual notions, while in hoop, with the lack of a suitable algebraic addition, the focus is shifted to deductive systems also called filters. So the notion of ideals is missing in hoops. In [T], Aaly and et al., defined and characterized the notion of ideals and different kinds of ideals such as implicative, maximal and prime ideals on hoops. Then they investigated the relation between them and proved that every maximal implicative ideal of a $\vee$-hoop with (DNP) is a prime one. Also, they defined a congruence relation on hoops by ideals and studied the quotient that is made by it. This notion helped to show that an ideal is maximal if and only if the quotient hoop is a simple MV-algebra. Also, they investigated the relationship between ideals and filters by exploiting the set of complements. In addition, in [3], Aaly and et al., introduced the notions of annihilators in hoops, investigated some related properties of them and proved that annihilators are ideals of hoop. In addition, they showed that the set of all ideals of hoop is a bounded distributive pseudocomplement lattice, and by using this result, they proved that the set of all annihilators of hoop, is a Boolean algebra. Also, they used the notion of annihilator and introduced the special kind of ideal of hoop as $\alpha$-ideal and showed that the set of all $\alpha$-ideals of hoop is a complete distributive lattice and consequenced that under what condition it is a Boolean algebra. Now, in this paper, we define the notion of minimal prime ideals of hoops and investigate some properties of them. Then by using the notion of annihilators, we study the relation between minimal prime ideals and annihilators. Also, we introduce the notion of zero divisors elements of hoops and prove that the set of all zero divisors of hoops is a union of all minimal prime ideals of hoop. Finally, by using the notions of minimal prime ideals and maximal ideals of hoop, we introduce two new ideals as $p$-ideal and $m$-ideal. Then we study some properties of them and investigate the relation between them and prove that every $p$-ideal of semi-simple hoop is an $m$-ideal of it.

\section{Preliminaries}

In this section, we gather some basic notions relevant to hoop which will need in the next sections.

A hoop is an algebraic structure $(\mathcal{H}, \odot, \rightarrow, 1)$ of type $(2,2,0)$ such that, for all $x, y, z \in \mathcal{H}$ :

$(H P 1) \quad(\mathcal{H}, \odot, 1)$ is a commutative monoid,

$(H P 2) \quad x \rightarrow x=1$,

$(H P 3) \quad(x \odot y) \rightarrow z=x \rightarrow(y \rightarrow z)$,

$(H P 4) \quad x \odot(x \rightarrow y)=y \odot(y \rightarrow x)$.

On hoop $\mathcal{H}$ we define $x \leq y$ if and only if $x \rightarrow y=1$. Obviously $(\mathcal{H}, \leq)$ is a poset. A bounded hoop $\mathcal{H}$ is a hoop which has the least element such as 0 such that $0 \leq x$, for all $x \in \mathcal{H}$. We let $x^{0}=1, x^{n}=x^{n-1} \odot x$, for any $n \in \mathbb{N}$. Let $\mathcal{H}$ be a bounded hoop. Define a unary operation ' on $\mathcal{H}$ by, $x^{\prime}=x \rightarrow 0$, for all $x \in \mathcal{H}$. If $\left(x^{\prime}\right)^{\prime}=x$, for all $x \in \mathcal{H}$, then the bounded hoop $\mathcal{H}$ is said to have the double negation property, or (DNP) for short. 
Proposition 2.1. [6, [ $\mathbb{7}, \mathbb{8}]$ Let $(\mathcal{H}, \odot, \rightarrow, 1)$ be a hoop. Then for all $x, y, z \in \mathcal{H}$ we have:

(i) $(\mathcal{H}, \leq)$ is a $\wedge$-semilattice with $x \wedge y=x \odot(x \rightarrow y)$,

(ii) $x \odot y \leq z$ if and only if $x \leq y \rightarrow z$,

(iii) $x \odot y \leq x, y$,

(iv) $x \rightarrow 1=1$ and $1 \rightarrow x=x$,

(v) $x \odot(x \rightarrow y) \leq y$,

(vi) $x \leq y$ implies $x \odot z \leq y \odot z, z \rightarrow x \leq z \rightarrow y$ and $y \rightarrow z \leq x \rightarrow z$,

(vii) $x \rightarrow(y \wedge z)=(x \rightarrow y) \wedge(x \rightarrow z)$.

(viii) If $\mathcal{H}$ is bounded, then $x \leq x^{\prime \prime}, x \odot x^{\prime}=0, x^{\prime \prime \prime}=x^{\prime}$ and $x \leq x^{\prime} \rightarrow y$.

Proposition 2.2. [ [8] Let $\mathcal{H}$ be a hoop and for any $x, y \in \mathcal{H}$, we define, $x \sqcup y=((x \rightarrow y) \rightarrow$ $y) \wedge((y \rightarrow x) \rightarrow x)$. Then the following conditions are equivalent:

(i) $\sqcup$ is an associative operation on $\mathcal{H}$,

(ii) $x \leq y$ implies $x \sqcup z \leq y \sqcup z$, for all $x, y, z \in \mathcal{H}$,

(iii) $\quad x \sqcup(y \wedge z) \leq(x \sqcup y) \wedge(x \sqcup z)$, for all $x, y, z \in \mathcal{H}$,

(iv) $\sqcup$ is the join operation on $\mathcal{H}$.

$A$ hoop $\mathcal{H}$ is called $a \sqcup$-hoop if $\sqcup$ is a join operation on $A$ and $\sqcup$-hoop is a distributive lattice.

Proposition 2.3. [8] Let $\mathcal{H}$ be $a \sqcup$-hoop. Then, for all $x, y, z \in \mathcal{H}$ we have:

(i) $\quad(x \sqcup y) \rightarrow z=(x \rightarrow z) \wedge(y \rightarrow z)$,

(ii) $\quad x \odot(y \sqcup z)=(x \odot y) \sqcup(x \odot z)$.

Note. From now on, we suppose $\mathcal{H}=(\mathcal{H}, \odot, \rightarrow, 0,1)$ or $\mathcal{H}$ is a bounded hoop unless otherwise state. Let $I$ be a non-empty subset of $\mathcal{H}$. Then $I$ is called an ideal of $\mathcal{H}$ if it satisfies the following conditions:

(I1) $0 \in I$,

(I2) for any $x, y \in I, x \ominus y=x^{\prime} \rightarrow y \in I$,

(I3) for any $x, y \in \mathcal{H}, x \leq y$ and $y \in I$ imply $x \in I$.

Obviously, $\mathcal{H}$ and $\{0\}$ are the trivial ideals of $\mathcal{H}$. The set of all ideals of $\mathcal{H}$ is denoted by $\mathcal{I} d(\mathcal{H})$. An ideal $I$ is called proper if $I \neq \mathcal{H}$. Clearly, an ideal $I$ is proper if and only if $1 \notin I$ (see [T]). Let $\emptyset \neq X \subseteq \mathcal{H}$. We recall that the smallest ideal containing $X$ in $\mathcal{H}$ is called the generated ideal by $X$ in $\mathcal{H}$ and it is denoted by $(X]$. It is also the intersection of all ideals of $\mathcal{H}$ containing $X$.

Theorem 2.4. [I] Let $\emptyset \neq X \subseteq \mathcal{H}$. Then

$(X]=\left\{a \in \mathcal{H} \mid \exists n \in \mathbb{N}\right.$ such that for $\left.x_{1}, x_{2}, \ldots, x_{n} \in X, a \leq x_{1} \ominus\left(x_{2} \ominus\left(\ldots \ominus\left(x_{n-1} \ominus x_{n}\right) \ldots\right)\right)\right\}$.

Note. Consider $a \ominus a \ominus \ldots \ominus a=n a=\left(a^{\prime}\right)^{n-1} \rightarrow a$. If $\mathcal{H}$ has (DNP), then $x \ominus y=y \ominus x$ and $n a=\left(\left(a^{\prime}\right)^{n}\right)^{\prime}$.

Proposition 2.5. [I] Let $I \in \mathcal{I} d(\mathcal{H})$ and $a \in \mathcal{H}$. Then the following statements hold,

(i) $\quad(a]=\{x \in \mathcal{H} \mid \exists n \in \mathbb{N}$ such that $x \leq n a\}$,

(ii) if $\mathcal{H}$ is a hoop with $(D N P)$, then $(I \cup\{a\}]=\left\{x \in \mathcal{H} \mid \exists n \in \mathbb{N}\right.$ such that $\left.x \odot(n a)^{\prime} \in I\right\}$,

(iii) if $\mathcal{H}$ is a $\sqcup$-hoop with (DNP), then $(I \cup\{x\}] \cap(I \cup\{y\}]=(I \cup\{x \wedge y\}]$.

Let $P$ be a proper ideal of $\mathcal{H}$. Then $P$ is called a prime ideal of $\mathcal{H}$ if $x \wedge y \in P$ implies $x \in P$ or $y \in P$, for any $x, y \in \mathcal{H}$. The set of all prime ideals of $\mathcal{H}$ is denoted by $\operatorname{Spec}(\mathcal{H})$. Let $U$ be a proper ideal of $\mathcal{H}$. Then $U$ is called a maximal ideal of $\mathcal{H}$ if no proper ideal of $\mathcal{H}$ strictly containing $U$. It means that if there exists an ideal of $\mathcal{H}$ such as $J$ that $U \subseteq J \subseteq \mathcal{H}$, then $U=J$ or $J=\mathcal{H}$. The set of all maximal ideals of $\mathcal{H}$ is denoted by $\operatorname{Max}(\mathcal{H})$ (see [T]). 
Theorem 2.6. [I] Let $\mathcal{H}$ be $a \sqcup$-hoop with (DNP), I be a proper ideal of $\mathcal{H}$ and $\emptyset \neq S \subseteq \mathcal{H}$ such that $I \cap S=\emptyset$. If $S$ is $\wedge$-closed, then there exists $P \in \mathcal{S p e c}(\mathcal{H})$ such that $I \subseteq P$ and $P \cap S=\emptyset$.

Theorem 2.7. [T] Let $\mathcal{H}$ be a ப-hoop with $(D N P)$. Then $\mathcal{M a x}(\mathcal{H}) \subseteq \operatorname{Spec}(\mathcal{H})$.

Theorem 2.8. [I] Let $\mathcal{H}$ be $a \sqcup$-hoop with (DNP) and $P$ be a proper ideal of $\mathcal{H}$. Then $P$ is a prime ideal of $\mathcal{H}$ if and only if, for any $I, J \in \mathcal{I} d(\mathcal{H})$ such that $I \cap J \subseteq P$, we get $I \subseteq P$ or $J \subseteq P$.

Definition 2.9. [3] Let $X$ be a non-void subset of $\mathcal{H}$ and set $X^{\perp}=\{a \in \mathcal{H} \mid a \wedge x=0$, for any $x \in$ $X\}$. Then $X^{\perp}$ is called an annihilator of $X$.

Proposition 2.10. [3] Let $X$ be a non-empty subset of $\mathcal{H}$. Then $X^{\perp}$ is a proper ideal of $\mathcal{H}$.

\section{Minimal prime ideals on hoops}

In this section, we define the notion of minimal prime ideals and zero divisors elements of hoops and investigate some properties of them. Then by using the notion of annihilators, we study the relation between minimal prime ideals and annihilators. Also, we prove that the set of all zero divisors of hoops is a union of all minimal prime ideals of hoop. But before that we need some properties about prime ideals and we prove them.

Theorem 3.1. Every proper ideal of $\mathcal{H}$ is contained in a maximal ideal of $\mathcal{H}$.

Proof. Suppose $I$ is a proper ideal of $\mathcal{H}$. Define

$$
\sum=\{Q \in \mathcal{I} d(\mathcal{H}) \mid Q \text { is a proper ideal of } \mathcal{H} \text { such that } I \subseteq Q\} .
$$

Since $I \in \sum$, we have $\sum \neq \emptyset$. Consider $\left(\sum, \subseteq\right)$. If $\left\{Q_{i}\right\}_{i \in I}$ is a family of proper ideals of $\mathcal{H}$ containing $I$, then by Zorn's Lemma, $U=\bigcup_{i \in I} Q_{i}$ is a maximal element of $\sum$. Clearly, $U$ is a maximal ideal of $\mathcal{H}$ containing $I$. Because if there exists a proper ideal $J$ of $\mathcal{H}$ such that $U \subseteq J$, then $I \subseteq J$ and so $J \in \sum$ which is a contradiction with being maximal element $U$. Therefore, every proper ideal of $\mathcal{H}$ is contained in a maximal ideal of $\mathcal{H}$.

Proposition 3.2. If $\mathcal{H}$ is a chain, then every proper ideal of $\mathcal{H}$ is prime. In addition, $\{0\} \in$ $\operatorname{Spec}(\mathcal{H})$.

Proof. Suppose $P$ is a proper ideal of $\mathcal{H}$ such that $x \wedge y \in P$, for $x, y \in \mathcal{H}$. By assumption, $\mathcal{H}$ is a chain, thus $x \leq y$ or $y \leq x$. If $x \leq y$, then $x=x \wedge y \in P$. By the similar way, if $y \leq x$, then $y \in P$. Hence, $P \in \operatorname{Spec}(\mathcal{H})$. In addition, $\{0\}$ is a proper ideal of $\mathcal{H}$, hence $\{0\} \in \mathcal{S p e c}(\mathcal{H})$.

Proposition 3.3. Let $\mathcal{H}$ be $a \sqcup$-hoop with (DNP). Then

(i) $\bigcap\{P \mid P \in \operatorname{Spec}(\mathcal{H})\}=\{0\}$.

(ii) If $0 \neq x \in \mathcal{H}$, then there exists $P \in \mathcal{S p e c}(\mathcal{H})$ such that $x \notin P$.

Proof. (i) Clearly, $\{0\} \subseteq \bigcap\{P \mid P \in \operatorname{Spec}(\mathcal{H})\}$. Suppose $0 \neq x \in \bigcap\{P \mid P \in \operatorname{Spec}(\mathcal{H})\}$. Consider $I=\{0\}$ and $S=\{x\}$. Then by Theorem [2.6, there exists $P \in \mathcal{S p e c}(\mathcal{H})$ such that $I \subseteq P$ and $x \notin P$, which is a contradiction. Therefore, $x=0$, and so $\bigcap\{P \mid P \in \mathcal{S p e c}(\mathcal{H})\}=\{0\}$.

(ii) Let $0 \neq x \in \mathcal{H}$ such that for all $P \in \mathcal{S p e c}(\mathcal{H}), x \in P$. Thus by (i), we have $x \in \bigcap\{P \mid P \in$ $\operatorname{Spec}(\mathcal{H})\}=\{0\}$, and so $x=0$, which is a contradiction.

Theorem 3.4. Let $I \in \mathcal{I} d(\mathcal{H})$ and $P_{i} \in \mathcal{S p e c}(\mathcal{H})$, for $1 \leq i \leq n$ such that $I \subseteq \bigcup_{i=1}^{n} P_{i}$. Then there exists $P_{i}$, for $1 \leq i \leq n$, such that $I \subseteq P_{i}$. 
Proof. We prove it by induction on $n$. If $n=2$, then $I \subseteq P_{1} \cup P_{2}$. We show that $I \subseteq P_{1}$ or $I \subseteq P_{2}$. Suppose $I \nsubseteq P_{1}$ and $I \nsubseteq P_{2}$. Then there exist $x_{2} \in I \backslash P_{1}$ and $x_{1} \in I \backslash P_{2}$. Since $I \subseteq P_{1} \cup P_{2}$, we have $x_{1} \in P_{1}$ and $x_{2} \in P_{2}$. Moreover, from $I \in \mathcal{I} d(\mathcal{H})$, we get $x_{1} \ominus x_{2} \in I \subseteq P_{1} \cup P_{2}$. We have the following cases:

Case 1. If $x_{1} \ominus x_{2} \in P_{1}$, since $x_{2} \leq x_{1} \ominus x_{2}$, we get $x_{2} \in P_{1}$, which is a contradiction.

Case 2. If $x_{1} \ominus x_{2} \in P_{2}$, since $x_{1} \leq x_{1} \ominus x_{2}$, we get $x_{1} \in P_{2}$, which is a contradiction.

Hence, $x_{1} \ominus x_{2} \notin P_{1} \cup P_{2}$, that is a contradiction. Therefore, $I \subseteq P_{1}$ or $I \subseteq P_{2}$. Suppose that the theorem holds for $n=k$. We prove that it holds for $n=k+1$. For this, let $I \subseteq \bigcup_{i=1}^{k+1} P_{i}$. If for any $1 \leq i \leq k+1$, we have $I \nsubseteq P_{i}$, then there exists $x_{j} \in I \backslash \bigcup_{i=1, i \neq j}^{k+1} P_{i}$ such that $x_{j} \in P_{j}$ and $x_{j} \notin \bigcap_{i=1, i \neq j}^{k+1} P_{i}$. Thus $x_{1} \wedge x_{2} \wedge \ldots \wedge x_{k} \notin P_{k+1}$, and so $x_{1} \wedge x_{2} \wedge \ldots \wedge x_{k} \in \bigcap_{i=1}^{k} P_{i} \backslash P_{k+1}$ and $x_{k+1} \in P_{k+1} \backslash \bigcup_{i=1}^{k} P_{i}$. Now, let $y=\left(x_{1} \wedge x_{2} \wedge \ldots \wedge x_{k}\right) \ominus x_{k+1}$. From $x_{j} \in I \backslash \bigcup_{i=1, i \neq j}^{k+1} P_{i}$ and $I \in$ $\mathcal{I} d(\mathcal{H})$, we have $y \in I$. If $y \in P_{k+1}$, then since $x_{1} \wedge x_{2} \wedge \ldots \wedge x_{k} \leq y$, we get $x_{1} \wedge x_{2} \wedge \ldots \wedge x_{k} \in P_{k+1}$, which is a contradiction, and so $y \notin P_{k+1}$. Since $I \subseteq \bigcup_{i=1}^{k+1} P_{i}$ and $y \notin P_{k+1}$, we obtain $y \in \bigcup_{i=1}^{k} P_{i}$. Then by assumption of induction, there exists $1 \leq i \leq k$ such that $I \subseteq P_{i}$. This complete the proof.

Lemma 3.5. Suppose $\mathcal{H}$ has (DNP). Then for any $x, y, z \in \mathcal{H}$ we have $x \wedge(y \ominus z) \leq(x \wedge y) \ominus(x \wedge z)$.

Proof. Let $x, y, z \in \mathcal{H}$. Then

$$
\begin{aligned}
& {[x \wedge(y \ominus z)] \rightarrow[(x \wedge y) \ominus(x \wedge z)]} \\
& =\left[x \wedge\left(y^{\prime} \rightarrow z\right)\right] \rightarrow\left[(x \wedge y)^{\prime} \rightarrow(x \wedge z)\right] \text { by (HP3) } \\
& =(x \wedge y)^{\prime} \rightarrow\left[\left(x \wedge\left(y^{\prime} \rightarrow z\right)\right) \rightarrow(x \wedge z)\right] \text { by Proposition } \mathbb{L} \text { (vii) } \\
& =(x \wedge y)^{\prime} \rightarrow\left[\left(\left(x \wedge\left(y^{\prime} \rightarrow z\right)\right) \rightarrow x\right) \wedge\left(\left(x \wedge\left(y^{\prime} \rightarrow z\right)\right) \rightarrow z\right)\right] \\
& =(x \wedge y)^{\prime} \rightarrow\left[\left(\left(x \wedge\left(y^{\prime} \rightarrow z\right)\right) \rightarrow z\right)\right] \text { by (HP3) } \\
& =\left(\left(x \wedge\left(y^{\prime} \rightarrow z\right)\right) \rightarrow\left[(x \wedge y)^{\prime} \rightarrow z\right)\right] \quad \text { by }(\mathrm{DNP}) \\
& =\left(x \wedge\left(y^{\prime} \rightarrow z\right)\right) \rightarrow\left[(x \wedge y)^{\prime} \rightarrow z^{\prime \prime}\right] \text { by (HP3) } \\
& =\left(x \wedge\left(y^{\prime} \rightarrow z\right)\right) \rightarrow\left[z^{\prime} \rightarrow(x \wedge y)^{\prime \prime}\right] \quad \text { by }(\mathrm{DNP})
\end{aligned}
$$

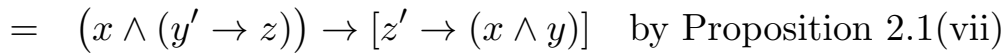

$$
\begin{aligned}
& =\left(x \wedge\left(y^{\prime} \rightarrow z\right)\right) \rightarrow\left[\left(z^{\prime} \rightarrow x\right) \wedge\left(z^{\prime} \rightarrow y\right)\right] \text { by Proposition }[.] \text { (vii) } \\
& =\left[\left(x \wedge\left(y^{\prime} \rightarrow z\right)\right) \rightarrow\left(z^{\prime} \rightarrow x\right)\right] \wedge\left[\left(x \wedge\left(y^{\prime} \rightarrow z\right)\right) \rightarrow\left(z^{\prime} \rightarrow y\right)\right] \text { by (HP3) } \\
& =\left[z^{\prime} \rightarrow\left(\left(x \wedge\left(y^{\prime} \rightarrow z\right)\right) \rightarrow x\right)\right] \wedge\left[\left(x \wedge\left(y^{\prime} \rightarrow z\right)\right) \rightarrow\left(z^{\prime} \rightarrow y^{\prime \prime}\right)\right] \quad \text { by (DNP) } \\
& =\left(x \wedge\left(y^{\prime} \rightarrow z\right)\right) \rightarrow\left(y^{\prime} \rightarrow z\right) \\
& =1 \text {. }
\end{aligned}
$$

Therefore, $x \wedge(y \ominus z) \leq(x \wedge y) \ominus(x \wedge z)$.

Proposition 3.6. Let $\mathcal{H}$ has $(D N P)$ and $P \in \mathcal{S p e c}(\mathcal{H})$. Then

$$
I_{P}=\{x \in \mathcal{H} \mid \text { there exists } y \notin P \text { such that } x \wedge y=0\},
$$

is a proper ideal of $\mathcal{H}$ and $I_{P} \subseteq P$.

Proof. For any $y \notin P, 0 \wedge y=0$, and so $0 \in I_{P}$. Suppose $a \in \mathcal{H}$ and $b \in I_{P}$ such that $a \leq b$. Then there is $y \notin P$ such that $b \wedge y=0$. Since $a \leq b$, we have $a \wedge y \leq b \wedge y=0$, and so $a \wedge y=0$. 
Hence, $a \in I_{P}$. Now, suppose $a, b \in I_{P}$. Then there are $x, y \notin P$ such that $a \wedge y=b \wedge x=0$. Since $P \in \mathcal{S p e c}(\mathcal{H})$, it is clear that $x \wedge y \notin P$. Thus

$$
\begin{aligned}
(a \ominus b) \wedge(y \wedge x) & =((a \ominus b) \wedge y) \wedge x \quad \text { by Lemma } 3.5 \\
& \leq((a \wedge y) \ominus(b \wedge y)) \wedge x \\
& =(0 \ominus(b \wedge y)) \wedge x=(b \wedge y) \wedge x=0
\end{aligned}
$$

Hence, $(a \ominus b) \wedge(y \wedge x)=0$, and so $a \ominus b \in I_{P}$. Hence, $I_{P} \in \mathcal{I} d(\mathcal{H})$. In addition, obviously $1 \notin I_{P}$, and so $I_{P}$ is a proper ideal of $\mathcal{H}$. Let $x \in I_{P}$. Then there exists $y \notin P$ such that $x \wedge y=0$. Since $P \in \mathcal{S} \operatorname{pec}(\mathcal{H}), y \notin P$ and $x \wedge y=0 \in P$, we get $x \in P$. Therefore, $I_{P} \subseteq P$.

Definition 3.7. An ideal $P$ of $\mathcal{H}$ is called a minimal prime ideal of $\mathcal{H}$ if $\left(M P_{1}\right) P \in \operatorname{Spec}(\mathcal{H})$.

$\left(M P_{2}\right)$ If there exists $Q \in \mathcal{S p e c}(\mathcal{H})$ such that $Q \subseteq P$, then $Q=P$.

The set of all minimal prime ideals of $\mathcal{H}$ is denoted by $\mathcal{M i n}(\mathcal{H})$.

Example 3.8. Let $\mathcal{H}=\{0, a, b, 1\}$ be a set by following Hasse diagram. Define the operations $\odot$ and $\rightarrow$ on $\mathcal{H}$ as follows:

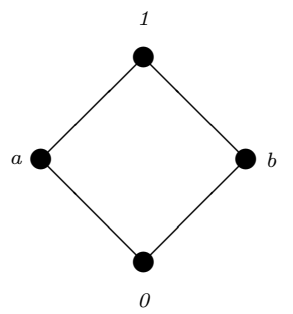

\begin{tabular}{l|llll}
$\odot$ & 0 & $a$ & $b$ & 1 \\
\hline 0 & 0 & 0 & 0 & 0 \\
$a$ & 0 & $a$ & 0 & $a$ \\
$b$ & 0 & 0 & $b$ & $b$ \\
1 & 0 & $a$ & $b$ & 1
\end{tabular}

\begin{tabular}{l|llll}
$\rightarrow$ & 0 & $a$ & $b$ & 1 \\
\hline 0 & 1 & 1 & 1 & 1 \\
$a$ & $b$ & 1 & $b$ & 1 \\
$b$ & $a$ & $a$ & 1 & 1 \\
1 & 0 & $a$ & $b$ & 1
\end{tabular}

Then $(\mathcal{H}, \odot, \rightarrow, 0,1)$ is a bounded hoop. Clearly, $\mathcal{I} d(\mathcal{H})=\{\{0\},\{0, a\},\{0, b\}, \mathcal{H}\}$ and $\mathcal{M i n}(\mathcal{H})=$ $\{\{0, a\},\{0, b\}\}$. Obviously, $\{0\} \notin \mathcal{S p e c}(\mathcal{H})$.

Note. Every minimal prime ideal of $\mathcal{H}$ is prime.

Corollary 3.9. Let $\mathcal{H}$ be $a \sqcup$-hoop with (DNP). Then

(i) $\bigcap\{P \mid P \in \mathcal{M i n}(\mathcal{H})\}=\{0\}$.

(ii) If $0 \neq x \in \mathcal{H}$, then there exists $P \in \mathcal{M i n}(\mathcal{H})$ such that $x \notin P$.

Proof. Since every minimal prime ideal of $\mathcal{H}$ is prime ideal, by Proposition $[3.3$ the proof is clear.

Theorem 3.10. Let $I$ be a proper ideal of $\mathcal{H}$ and $P \in \mathcal{S p e c}(\mathcal{H})$ such that $I \subseteq P$. Then there is $Q \in \mathcal{M i n}(\mathcal{H})$ such that $Q \subseteq P$.

Proof. Let $\sum=\{U \in \operatorname{Spec}(\mathcal{H}) \mid I \subseteq U \subseteq P\}$. Since $P \in \sum$, we have $\sum \neq \emptyset$. Define $\ll$ on $\sum$ by $U_{1} \ll U_{2}$ if and only if $U_{2} \subseteq U_{1}$. Clearly, $\left(\sum, \ll\right)$ is a poset. Suppose $\left\{U_{i}\right\}_{i \in I}$ is a family of prime ideals of $\mathcal{H}$ such that for any $i \in I, U_{i} \in \sum$. Set $Q=\bigcap_{i \in I} U_{i}$. Obviously, $Q$ is a proper ideal of $\mathcal{H}$ which is true in terms of the set $\sum$. It is enough to prove that $Q$ is prime. For this suppose $x \wedge y \in Q$ such that $x \notin Q$. Then there exists $i \in I$ such that $x \notin U_{i}$. Suppose $U_{j} \in \sum$ such that $j \neq i$. We have the following cases:

Case 1. If $U_{j} \subseteq U_{i}$, then from $x \notin U_{i}$, we have $x \notin U_{j}$ where $x \wedge y \in Q \subseteq U_{j}$. Thus $y \in U_{j}$, and so for any $j \in I, y \in U_{j}$. Hence, $y \in Q$, and so $Q \in \mathcal{S p e c}(\mathcal{H})$.

Case 2. If $U_{i} \subseteq U_{j}$, then since $x \notin U_{i}$ and $U \in \mathcal{S p e c}(\mathcal{H})$ such that $x \wedge y \in Q \subseteq U_{j}$, we have $y \in U_{i}$. Thus for any $j \in I, y \in U_{j}$ and so $y \in Q$. Hence, $Q \in \mathcal{S p e c}(\mathcal{H})$. 
Thus, $Q$ is an upper bound of $\sum$ and by Zorn's Lemma, $\sum$ has a maximal element such as $Q^{*}$. Now, we prove that $Q^{*} \in \mathcal{M i n}(\mathcal{H})$. For this, suppose there is $\widehat{P} \in \mathcal{S p e c}(\mathcal{H})$ such that $I \subseteq \widehat{P} \subseteq Q^{*}$. Then $\widehat{P} \in \sum$ and $Q^{*} \ll \widehat{P}$, which is a contradiction. Therefore, $Q^{*} \in \mathcal{M i n}(\mathcal{H})$ such that $Q^{*} \subseteq P$.

Theorem 3.11. Let $\mathcal{H}$ be $a \sqcup$-hoop with $(D N P)$ and $P \in \mathcal{S p e c}(\mathcal{H})$. Then $P \in \mathcal{M i n}(\mathcal{H})$ if and only if for each $x \in P$, there exists $r \in \mathcal{H} \backslash P$ such that $x \wedge r=0$.

Proof. $(\Rightarrow)$ Suppose there exists $x \in P$ such that for any $r \in \mathcal{H} \backslash P$, we have $r \wedge x \neq 0$. Define $S=\{x \wedge r \mid r \in \mathcal{H} \backslash P\}$. Let $x \wedge r_{1}, x \wedge r_{2} \in S$, for $r_{1}, r_{2} \in \mathcal{H} \backslash P$. Then $\left(x \wedge r_{1}\right) \wedge\left(x \wedge r_{2}\right)=x \wedge\left(r_{1} \wedge r_{2}\right)$. If $r_{1} \wedge r_{2} \in P$, then since $P \in \mathcal{S p e c}(\mathcal{H})$, we get $r_{1} \in P$ or $r_{2} \in P$, which is a contradiction. Thus, $r_{1} \wedge r_{2} \in \mathcal{H} \backslash P$, and so $\left(x \wedge r_{1}\right) \wedge\left(x \wedge r_{2}\right) \in S$. Hence, $S$ is $\wedge$-closed. By Theorem [2.6, there exists $Q \in \operatorname{Spec}(\mathcal{H})$ such that $S \cap Q=\emptyset$. Now, we have two following cases:

Case 1. If $Q \subseteq P$, then by assumption, since $P \in \mathcal{M i n}(\mathcal{H})$, we get $Q=P$ and so $P \cap S=\emptyset$. But by hypothesis, $x \in P, 1 \in \mathcal{H} \backslash P$, and $x=1 \wedge x \in S$, and so $P \cap S \neq \emptyset$, which is a contradiction.

Case 2. If $Q \nsubseteq P$, then there exists $r \in Q \backslash P$, and so $r \in \mathcal{H} \backslash P$. Thus $x \wedge r \in S$. Also, since $Q \in \operatorname{Spec}(\mathcal{H})$ and $x \wedge r \leq r$, we get $x \wedge r \in Q$. Hence, $x \wedge r \in Q \cap S \neq \emptyset$, which is a contradiction. Since in both cases we have a contradiction, we consequence that for each $x \in P$, there exists $r \in \mathcal{H} \backslash P$ such that $x \wedge r=0$.

$(\Leftarrow)$ Suppose that there exists $Q \in \mathcal{S p e c}(\mathcal{H})$ such that $Q \subseteq P$. If $Q \neq P$, then there is $x \in P \backslash Q$. By assumption, there exists $r \in \mathcal{H} \backslash P$ such that $x \wedge r=0$. Since $Q \in \mathcal{S} p e c(\mathcal{H}), x \wedge r=0 \in Q$ and $x \notin Q$, we have $r \in Q$. Also, from $Q \subseteq P$ we get $r \in P$, which is a contradiction. Therefore, $Q=P$, and so $P \in \mathcal{M i n}(\mathcal{H})$.

Corollary 3.12. Let $\mathcal{H}$ be $a \sqcup$-hoop with (DNP). If $P \in \mathcal{M i n}(\mathcal{H})$, then for any $x \in P$ there is $y \in \mathcal{H} \backslash P$ and $k \in \mathbb{N}$ such that $y \wedge k n=0$.

Theorem 3.13. Let $\mathcal{H}$ be $a \sqcup$-hoop with (DNP), $P \in \mathcal{M i n}(\mathcal{H})$ and $I$ be a finitly generated ideal of $\mathcal{H}$. Then $I \subseteq P$ if and only if $I^{\perp} \nsubseteq P$.

Proof. $(\Rightarrow)$ By assumption, $I$ is a finitly generated ideal of $\mathcal{H}$. Then there exist $a_{1}, a_{2}, \ldots, a_{n} \in \mathcal{H}$ such that $I=\left(a_{1}, a_{2}, \ldots, a_{n}\right]$. Thus, for any $x \in I$, we have $x \leq a_{1} \ominus\left(a_{2} \ominus\left(\ldots \ominus\left(a_{n-1} \ominus a_{n}\right) \ldots\right)\right)$. Since $I \subseteq P$, for any $1 \leq i \leq n, a_{i} \in P$ and by Theorem $[$.$] , there exist u_{i} \in \mathcal{H} \backslash P$ such that $a_{i} \wedge u_{i}=0$. Set $u=\bigwedge_{i=1}^{n} u_{i}$. Clearly $u \notin P$. Then by Lemma $\mathbf{3 . 5}$, we have

$$
u \wedge x \leq u \wedge\left[a_{1} \ominus\left(a_{2} \ominus\left(\ldots \ominus\left(a_{n-1} \ominus a_{n}\right) \ldots\right)\right)\right] \leq\left(u \wedge a_{1}\right) \ominus\left(u \wedge a_{2}\right) \ominus \ldots \ominus\left(u \wedge a_{n}\right)=0 .
$$

Thus $u \wedge x=0$, and so $u \in I^{\perp}$. Hence, there is an element $u \in I^{\perp}$ such that $u \notin P$. Therefore, $I^{\perp} \nsubseteq P$.

$(\Leftarrow)$ Suppose $I^{\perp} \nsubseteq P$. Then there exists $x \in I^{\perp}$ such that $x \notin P$. Since $x \in I^{\perp}$, for any $y \in I$, $x \wedge y=0$. From $P \in \mathcal{M i n}(\mathcal{H}), x \wedge y=0 \in P$ and $x \notin P$, we have $y \in P$. Hence, $I \subseteq P$.

Proposition 3.14. Let $\mathcal{H}$ be $a \sqcup$-hoop with $(D N P)$ and $P \in \mathcal{S p e c}(\mathcal{H})$. If $I_{P}=P$, then $P \in$ $\operatorname{Min}(\mathcal{H})$.

Proof. By Proposition 5.6 and Theorem [.]1, the proof is clear.

Definition 3.15. Let $X$ be a non-empty subset of $\mathcal{H}$. The set of all zero divisors of $X$ is denoted by $\mathcal{Z}_{X}(\mathcal{H})$ and defined as follows:

$$
\mathcal{Z}_{X}(\mathcal{H})=\{a \in \mathcal{H} \mid \text { there exists } 0 \neq x \in X \text { such that } x \wedge a=0\} .
$$

The set of all zero divisors of $\mathcal{H}$ is denoted by $\mathcal{Z}_{\mathcal{H}}$. 
Example 3.16. $(i)$ The zero element of $\mathcal{H}$ is a trivial zero divisor of $\mathcal{H}$.

(ii) Let $\mathcal{H}$ be the hoop as Example [., S. Suppose $X_{1}=\{a\}$ and $X_{2}=\{a, b\}$. Then $\mathcal{Z}_{X_{1}}(\mathcal{H})=\{0, b\}$ and $\mathcal{Z}_{X_{2}}(\mathcal{H})=\{0, a, b\}$.

Theorem 3.17. Let $\mathcal{H}$ be $a \sqcup$-hoop with (DNP). Then the set of all zero divisors of $\mathcal{H}$ contains at least one prime ideal of $\mathcal{H}$.

Proof. For proving this theorem, we prove that for $0 \neq x \in \mathcal{H} \backslash \mathcal{Z}_{\mathcal{H}}$, there exists $P \in \mathcal{S} p e c(\mathcal{H})$ such that $x \in P$ and $P \subseteq \mathcal{Z}_{\mathcal{H}}$. Let $x, y \notin \mathcal{Z}_{\mathcal{H}}$. Then for any $a, b \in \mathcal{H} \backslash\{0\}, x \wedge a \neq 0$ and $y \wedge b \neq 0$. If $x \wedge y \in \mathcal{Z}_{\mathcal{H}}$, then there is $u \in \mathcal{H}$ such that $(x \wedge y) \wedge u=0$, and so $x \wedge(y \wedge u)=0$ or $y \wedge(x \wedge u)=0$. Thus $x \in \mathcal{Z}_{\mathcal{H}}$ or $y \in \mathcal{Z}_{\mathcal{H}}$, which is a contradiction. Thus $x \wedge y \notin \mathcal{Z}_{\mathcal{H}}$, and so $\mathcal{H} \backslash \mathcal{Z}_{\mathcal{H}}$ is $\wedge$-closed. Let $x \in \mathcal{Z}_{\mathcal{H}}$. Then set $I=(x]$. We prove $I \subseteq \mathcal{Z}_{\mathcal{H}}$. Let $y \in I \backslash \mathcal{Z}_{\mathcal{H}}$. Then there is $n \in \mathbb{N}$ such that $y \leq n x$ and $y \notin \mathcal{Z}_{\mathcal{H}}$. Thus for any $a \in \mathcal{H} \backslash\{0\}, y \wedge a \neq 0$. Since $x \in \mathcal{Z}_{\mathcal{H}}$, by Definition [3.15, there is $0 \neq b \in \mathcal{H}$ such that $x \wedge b=0$. By Lemma [3.5, $y \wedge b \leq(n x) \wedge b \leq n(x \wedge b)=0$, and so $y \wedge b=0$, which is a contradiction. Thus $I \subseteq \mathcal{Z}_{\mathcal{H}}$. Since $\mathcal{H} \backslash \mathcal{Z}_{\mathcal{H}}$ is $\wedge$-closed and $I \cap\left(\mathcal{H} \backslash \mathcal{Z}_{\mathcal{H}}\right)=\emptyset$, by Theorem [2.6], there exists $P \in \mathcal{S p e c}(\mathcal{H})$ such that $(x]=I \subseteq P$ and $P \cap\left(\mathcal{H} \backslash \mathcal{Z}_{\mathcal{H}}\right)=\emptyset$ which means that $P \subseteq \mathcal{Z}_{\mathcal{H}}$.

Corollary 3.18. Let $\mathcal{H}$ be $a \sqcup$-hoop with (DNP). Then every element of a minimal prime ideal of $\mathcal{H}$ is zero divisor.

Proof. By Theorem 3.17 and Corollary 3.2 , the proof is clear.

In the following example we show that the converse of Theorem 3.17 does not hold.

Example 3.19. Let $\mathcal{H}$ be the hoop as in Example [3.8. Then $\mathcal{Z}_{\mathcal{H}}=\{0, a, b, 1\}=\mathcal{H}$ and clearly there is not any prime ideal containing $\mathcal{H}$.

Proposition 3.20. Let $\mathcal{H}$ be a $\sqcup$-hoop with (DNP). Then the set $\mathcal{Z}_{\mathcal{H}}$ is a union of all minimal prime ideals of $\mathcal{H}$.

Proof. We prove $\mathcal{Z}_{\mathcal{H}}=\bigcup_{P \in \mathcal{M i n}(\mathcal{H})} P$. Let $x \in \bigcup_{P \in \mathcal{M i n}(\mathcal{H})} P$. Then there is $P \in \operatorname{Min}(\mathcal{H})$ such that $x \in P$. By Corollary [.18, $x \in \mathcal{Z}_{\mathcal{H}}$ and so $\bigcup_{P \in \mathcal{M i n}(\mathcal{H})} P \subseteq \mathcal{Z}_{\mathcal{H}}$. Conversely, suppose $x \in \mathcal{Z}_{\mathcal{H}}$. Then there is $0 \neq y \in \mathcal{H}$ such that $x \wedge y=0$. Thus by Corollary $[\mathrm{g}$ (ii), there is $P \in \mathcal{M i n}(\mathcal{H})$ such that $y \notin P$. Since $P \in \operatorname{Spec}(\mathcal{H})$ and $x \wedge y=0 \in P$, we have $x \in P$. Hence, $\mathcal{Z}_{\mathcal{H}} \subseteq \bigcup_{P \in \mathcal{M i n}(\mathcal{H})} P$. Therefore, $\mathcal{Z}_{\mathcal{H}}$ is a union of all minimal prime ideals of $\mathcal{H}$.

Note. Define $P_{b}=\bigcap\{P \mid P \in \mathcal{M i n}(\mathcal{H})$ and $b \in P\}$ and $M_{b}=\bigcap\{M \mid M \in \mathcal{M a x}(\mathcal{H})$ and $b \in M\}$.

Theorem 3.21. Let $\mathcal{H}$ be $a \sqcup$-hoop with (DNP). If $x, y \in \mathcal{H}$ such that $y \in(x]^{\perp}$, then $(x]^{\perp} \subseteq P_{y}$ if and only if $x \ominus y \notin \mathcal{Z}_{\mathcal{H}}$.

Proof. $(\Rightarrow)$ Let $x, y \in \mathcal{H}$ such that $x \ominus y \in \mathcal{Z}_{\mathcal{H}}$. Then by Proposition $\mathbf{3 . 2 0}$, there exists $P \in \mathcal{M i n}(\mathcal{H})$ such that $x \ominus y \in P$. Since $y \leq x \ominus y$, we get $y \in P$. Thus $P y \subseteq P$. In addition, $x \leq x \ominus y$, and so $x \in P$. By assumption, $(x]^{\perp} \subseteq P$ and by Theorem [.13, $(x] \nsubseteq P$ and so $x \notin P$, which is a contradiction. Hence, $x \ominus y \notin \mathcal{Z}_{\mathcal{H}}$.

$(\Leftarrow)$ Suppose $(x]^{\perp} \nsubseteq P_{y}$. Then there is $a \in(x]^{\perp}$ such that $a \notin P_{y}$. Thus there is $P \in \mathcal{M i n}(\mathcal{H})$ such that $y \in P$ and $a \notin P$. Also, by Theorem [3.13, since $(x]^{\perp} \nsubseteq P_{y}$, we have $(x] \subseteq P$. Thus $x, y \in P$ and since $P$ is ideal, we have $x \ominus y \in P$. By Corollary [3.18, since $P \subseteq \mathcal{Z}_{\mathcal{H}}$, we get $x \ominus y \in \mathcal{Z}_{\mathcal{H}}$ which is a contradiction. Hence $(x]^{\perp} \subseteq P_{y}$. 
Theorem 3.22. Let $\mathcal{H}$ be $a \sqcup$-hoop with (DNP) and $x \in \mathcal{H}$. Then $P_{x}=\left\{y \in \mathcal{H} \mid x^{\perp} \subseteq y^{\perp}\right\}$.

Proof. Suppose $B=\left\{y \in \mathcal{H} \mid x^{\perp} \subseteq y^{\perp}\right\}$. Let $z \in P_{x}$ such that $z \notin B$. Since $z \notin B$, we get $x^{\perp} \nsubseteq z^{\perp}$. Then there exists $u \in x^{\perp}$ such that $u \notin z^{\perp}$. Thus $u \wedge z \neq 0$. By Corollary B.g, there exists $Q \in \mathcal{M i n}(\mathcal{H})$ such that $u \wedge z \notin Q$, and so $u \notin Q$ and $z \notin Q$. In addition, since $u \in x^{\perp}$, we have $u \wedge x=0 \in Q$, and so $x \in Q$. Hence, we find $Q \in \mathcal{M i n}(\mathcal{H})$ such that $x \in Q$ and $z \notin Q$. Thus $z \notin P_{x}$, which is a contradiction. Therefore, $P_{x} \subseteq B$. Conversely, suppose $y \in B$ such that $y \notin P_{x}$. Since $y \in B$, we have $x^{\perp} \subseteq y^{\perp}$. From $y \notin P_{x}$ we get that there is $Q \in \mathcal{M i n}(\mathcal{H})$ such that $x \in Q$ and $y \notin Q$. Thus $(y] \nsubseteq Q$. By Theorem [3.13, since $(y]$ is finite generated, $y^{\perp} \subseteq Q$. Also, from $x \in Q$, we have $(x] \subseteq Q$ and by Theorem [3.13, $x^{\perp} \nsubseteq Q$. Thus there exists $u \in x^{\perp}$ such that $u \notin Q$. Since $y^{\perp} \subseteq Q$, we get $u \notin y^{\perp}$, and so $x^{\perp} \nsubseteq y^{\perp}$, which is a contradiction. Hence, $B \subseteq P_{x}$. Therefore, $P_{x}=\left\{y \in \mathcal{H} \mid x^{\perp} \subseteq y^{\perp}\right\}$.

Definition 3.23. $(i)$ Let $I$ be a proper ideal of $\mathcal{H}$. Then $I$ is called a p-ideal of $\mathcal{H}$ if $P_{x} \subseteq I$, for any $x \in I$.

(ii) Let $I$ be a proper ideal of $\mathcal{H}$. Then $I$ is called an $m$-ideal of $\mathcal{H}$ if $M_{x} \subseteq I$, for any $x \in I$.

Example 3.24. $(i)$ Let $\mathcal{H}$ be the hoop as in Example [3.8. Then $\{0\}$ is a p-ideal of $\mathcal{H}$.

(ii) Let $\mathcal{H}=\{0, a, b, c, d, e, f, 1\}$. Define two operations $\odot$ and $\rightarrow$ on $\mathcal{H}$ as follows,

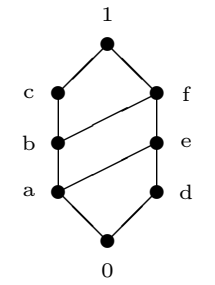

Figure 1: The Hasse diagram of $\mathcal{H}$

\begin{tabular}{l|llllllll}
$\rightarrow$ & 0 & $a$ & $b$ & $c$ & $d$ & $e$ & $f$ & 1 \\
\hline 0 & 1 & 1 & 1 & 1 & 1 & 1 & 1 & 1 \\
$a$ & $d$ & 1 & 1 & 1 & $d$ & 1 & 1 & 1 \\
$b$ & $d$ & $f$ & 1 & 1 & $d$ & $f$ & 1 & 1 \\
$c$ & $d$ & $e$ & $f$ & 1 & $d$ & $e$ & $f$ & 1 \\
$d$ & $c$ & $c$ & $c$ & $c$ & 1 & 1 & 1 & 1 \\
$e$ & 0 & $c$ & $c$ & $c$ & $d$ & 1 & 1 & 1 \\
$f$ & 0 & $b$ & $c$ & $c$ & $d$ & $f$ & 1 & 1 \\
1 & 0 & $a$ & $b$ & $c$ & $d$ & $e$ & $f$ & 1
\end{tabular}

\begin{tabular}{l|llllllll}
$\odot$ & 0 & $a$ & $b$ & $c$ & $d$ & $e$ & $f$ & 1 \\
\hline 0 & 0 & 0 & 0 & 0 & 0 & 0 & 0 & 0 \\
$a$ & 0 & $a$ & $a$ & $a$ & 0 & $a$ & $a$ & $a$ \\
$b$ & 0 & $a$ & $a$ & $b$ & 0 & $a$ & $a$ & $b$ \\
$c$ & 0 & $a$ & $b$ & $c$ & 0 & $a$ & $b$ & $c$ \\
$d$ & 0 & 0 & 0 & 0 & $d$ & $d$ & $d$ & $d$ \\
$e$ & 0 & $a$ & $a$ & $a$ & $d$ & $e$ & $e$ & $e$ \\
$f$ & 0 & $a$ & $a$ & $b$ & $d$ & $e$ & $e$ & $f$ \\
1 & 0 & $a$ & $b$ & $c$ & $d$ & $e$ & $f$ & 1
\end{tabular}

Then $(\mathcal{H}, \odot, \rightarrow, 0,1)$ is a bounded hoop. Then $\mathcal{I} d(\mathcal{H})=\{\{0\},\{0, d\},\{0, a, b, c\}, \mathcal{H}\}$ and $\mathcal{M i n}(\mathcal{H})=$ $\{\{0, d\},\{0, a, b, c\}\}$. Clearly, $\{0, d\}$ and $\{0, a, b, c\}$ are $p$-ideal and m-ideal of $\mathcal{H}$. Also, $P_{a}=$ $\{0, a, b, c\}$ and $P_{e}=\emptyset$.

Clearly, any arbitrary intersection of $p$-ideals of $\mathcal{H}$ is a $p$-ideal of $\mathcal{H}$.

Theorem 3.25. Let $\mathcal{H}$ be a ப-hoop with (DNP). If $I$ is a proper ideal of $\mathcal{H}$, then $I^{\perp}$ is a p-ideal of $\mathcal{H}$. 
Proof. Let $x \in I^{\perp}$ and $u \in P_{x}$. Then by Theorem [.22, $u \in\left\{y \in \mathcal{H} \mid x^{\perp} \subseteq y^{\perp}\right\}$. Thus $x^{\perp} \subseteq u^{\perp}$. If $u \notin I^{\perp}$, then $u \wedge I \neq 0$, and so $I \nsubseteq u^{\perp}$. Since $x^{\perp} \subseteq u^{\perp}$, we get $I \nsubseteq x^{\perp}$. Hence, $x \notin I^{\perp}$, which is a contradiction. Thus, $P_{x} \subseteq I^{\perp}$, for any $x \in I^{\perp}$. Therefore, $I^{\perp}$ is a $p$-ideal of $\mathcal{H}$.

Corollary 3.26. Let $\mathcal{H}$ be $a \sqcup$-hoop with $(D N P)$. If $I \in \mathcal{I} d(\mathcal{H})$, then $I^{\perp \perp}$ is a p-ideal of $\mathcal{H}$.

Proof. By Proposition [2.10, $I^{\perp}$ is a proper ideal of $\mathcal{H}$. Then by Theorem $[2.5$, the proof is clear.

Theorem 3.27. Let $\mathcal{H}$ be $a \sqcup$-hoop with (DNP). If I is a p-ideal of $\mathcal{H}$, then every element of $I$ is zero divisor.

Proof. Suppose $x \in I$. By assumption, $P_{x} \subseteq I$. Since $P_{x}=\bigcap\{P \mid P \in \mathcal{M i n}(\mathcal{H})$ such that $x \in P\}$, there is $P \in \mathcal{M i n}(\mathcal{H})$ such that $x \in P$. By Corollary $\mathbf{3 . 1 8}$, we get $x$ is zero divisor.

Proposition 3.28. Let $\mathcal{H}$ be $a \sqcup$-hoop with (DNP). Then for any $x \in \mathcal{H}, P_{x}=\left((x]^{\perp}\right)^{\perp}$.

Proof. Suppose $y \in\left((x]^{\perp}\right)^{\perp}$ such that $y \notin P_{x}$. Thus there is $P \in \mathcal{M i n}(\mathcal{H})$ such that $x \in P$ and $y \notin P$. Since $x \in P$, we have $(x] \subseteq P$ and by Theorem [.].3, $(x]^{\perp} \nsubseteq P$. Also, from $y \notin P$, we get $(y] \nsubseteq P$ and by Theorem [3.13, $(y]^{\perp} \subseteq P$. Moreover, since $y \in\left((x]^{\perp}\right)^{\perp}$, we get $y \wedge(x]^{\perp}=0 \in P$. Since $P \in \mathcal{M i n}(\mathcal{H})$, we get $y \in P$ or $(x]^{\perp} \subseteq P$, which is contradiction in bouth cases. Hence, $\left((x]^{\perp}\right)^{\perp} \subseteq P_{x}$. Conversely, suppose $y \in P_{x}$ such that $y \notin\left((x]^{\perp}\right)^{\perp}$. Since $y \in P_{x}$, we have for any $P \in \mathcal{M i n}(\mathcal{H})$ such that $x \in P$, we have $y \in P$. From $y \notin\left((x]^{\perp}\right)^{\perp}$, we have $y \wedge(x]^{\perp} \neq 0$. By Corollary $\operatorname{Bg}\left(\right.$ ii), there exists $P \in \mathcal{M i n}(\mathcal{H})$ such that $y \wedge(x]^{\perp} \notin P$. Thus $y \notin P$ and $(x]^{\perp} \nsubseteq P$. Since $(x]$ is finite generated, by Theorem 3.3 we have $(x] \subseteq P$, and so $x \in P$. Hence, we find $P \in \mathcal{M i n}(\mathcal{H})$ such that $x \in P$ and $y \notin P$, which is a contradiction. Thus $P \subseteq\left(x^{\perp}\right)^{\perp}$. Therefore, $P_{x}=\left((x]^{\perp}\right)^{\perp}$.

Proposition 3.29. Let $\mathcal{H}$ be a $\sqcup$-hoop with (DNP). We have:

(i) If $I$ is a $p$-ideal of $\mathcal{H}$, then every element of $I$ is a zero divisor.

(ii) $\{0\}$ is a $p$-ideal of $\mathcal{H}$.

(iii) Every $P \in \mathcal{M i n}(\mathcal{H})$ is a p-ideal of $\mathcal{H}$.

Proof. (i) Since $I$ is a $p$-ideal of $\mathcal{H}$, for any $x \in I$, we have $P_{x} \subseteq I$. Also, by definition of $P_{x}$, $x \in P_{x}$ and there is $P \in \mathcal{M i n}(\mathcal{H})$ such that $x \in P$. Also, by Corollary $\mathbf{3 . 1 8}, x$ is a zero divisor. Hence, every element of $I$ is a zero divisor.

(ii) By Corollary [3.9, the proof is straightforward.

(iii) Since $P \in \mathcal{M i n}(\mathcal{H})$, we know $P \in \mathcal{S p e c}(\mathcal{H})$. Let $x \in P$. By definition of $P_{x}$ we have $P_{x} \subseteq P$. Hence, $P$ is a $p$-ideal of $\mathcal{H}$.

Proposition 3.30. Let $\mathcal{H}$ be $a \sqcup$-hoop with (DNP). Suppose $I \in \mathcal{I} d(\mathcal{H})$ such that every element of $I$ is a zero divisor. Then there is a p-ideal of $\mathcal{H}$ containing $I$.

Proof. Let $x \in I$. Then by assumption, $x$ is a zero divisor element. Thus by Proposition 3.20 , $x \in \bigcup_{P_{i} \in \mathcal{M i n}(\mathcal{H})} P_{i}$. So, there exists $P_{i} \in \mathcal{M i n}(\mathcal{H})$ such that $x \in P_{i}$. Thus, for any $x \in I$, there exists $P_{i} \in \mathcal{M i n}(\mathcal{H})$ such that $x \in P_{i}$. Hence, $I \subseteq \bigcup_{P_{i} \in \mathcal{M} \text { in }(\mathcal{H})} P_{i}$. By Theorem 3.4, there exists $P_{i} \in \operatorname{Min}(\mathcal{H})$ such that $I \subseteq P_{i}$. Also, by Proposition $\operatorname{3.2.g(iii),~} P_{i}$ is a $p$-ideal of $\mathcal{H}$. Therefore, there is a $p$-ideal of $\mathcal{H}$ containing $I$.

Lemma 3.31. For any $x, y \in \mathcal{H}, P_{x} \cup P_{y} \subseteq P_{x \wedge y}$. 
Proof. Let $\alpha \in P_{x} \cup P_{y}$. Then $\alpha \in P_{x}$ or $\alpha \in P_{y}$. If $\alpha \in P_{x}$, then for any $P \in \mathcal{M i n}(\mathcal{H})$ such that $x \in P$ we have $\alpha \in P$. Since $P \in \mathcal{M i n}(\mathcal{H})$, for any $y \in \mathcal{H}, x \wedge y \in P$. Thus for any $P \in \mathcal{M i n}(\mathcal{H})$, $x \wedge y \in P$ and so $\alpha \in P_{x \wedge y}$. The proof of other case is similar. Therefore, $P_{x} \cup P_{y} \subseteq P_{x \wedge y}$.

Theorem 3.32. Let $\mathcal{H}$ be $a \sqcup$-hoop with (DNP). Suppose $I$ is a p-ideal of $\mathcal{H}$ and $S$ be a non-empty set of $\mathcal{H}$ such that $S \nsubseteq I$. Then $\{x \in \mathcal{H} \mid$ for any $s \in S, x \wedge s \in I\}$ is a p-ideal of $\mathcal{H}$.

Proof. Let $I$ be a $p$-ideal of $\mathcal{H}$ and $B=\{x \in \mathcal{H} \mid$ for any $s \in S, x \wedge s \in I\}$. Since $S \nsubseteq I$, there exists $s^{*} \in S$ such that $s^{*} \notin I$. Suppose $y \in B$. Then for any $s \in S, y \wedge s \in I$. Thus, $y \wedge s^{*} \in I$. Since $I$ is a $p$-ideal of $\mathcal{H}$, we have $P_{y \wedge s^{*}} \subseteq I$. By Lemma [3.3l, clearly $P_{s^{*}} \cap P_{y} \subseteq P_{s^{*} \wedge y}$, and so $P_{s^{*}} \cap P_{y} \subseteq I$. Then by Theorem [2.8, $P_{s^{*}} \subseteq I$ or $P_{y} \subseteq I$. If $P_{s^{*}} \subseteq I$, since $s^{*} \in P_{s^{*}}$, we get $s^{*} \in I$, which is a contradiction. Thus $P_{y} \subseteq I$. Also, for any $z \in I$ and $s \in S$, we have $z \wedge s \leq z$ and so $z \wedge s \in I$. Hence, $I \subseteq B$, and so $P_{y} \subseteq B$. Therefore, $\{x \in \mathcal{H} \mid$ for any $s \in S, x \wedge s \in I\}$ is $p$-ideal.

Theorem 3.33. (i) If $\mathcal{H}$ has just one maximal ideal, then $\mathcal{H}$ has only one $m$-ideal.

(ii) Every chain hoop has only one m-ideal.

Proof. Clearly, every chain hoop has just one maximal ideal, and by (i), the proof is clear.

Proposition 3.34. Let $\mathcal{H}$ be $a \sqcup$-hoop with (DNP). Then $\{0\}$ is an m-ideal of $\mathcal{H}$.

Proof. By Theorem [2.], $\operatorname{Max}(\mathcal{H}) \subseteq \mathcal{S} \operatorname{pec}(\mathcal{H})$. Then by Proposition [3.3, we have

$$
\bigcap\{M \mid M \in \mathcal{M a x}(\mathcal{H})\} \subseteq \bigcap\{P \mid P \in \mathcal{S p e c}(\mathcal{H})\}=\{0\} .
$$

Thus $\bigcap\{M \mid M \in \mathcal{M a x}(\mathcal{H})\}=\{0\}$. Clearly, $M_{0} \subseteq\{0\}$ and so $\{0\}$ is an $m$-ideal of $\mathcal{H}$.

Theorem 3.35. Let $\mathcal{H}$ be $a \sqcup$-hoop with (DNP). Suppose $I$ is an m-ideal of $\mathcal{H}$ and $S$ be a nonempty set of $\mathcal{H}$ such that $S \nsubseteq I$. Then $\{x \in \mathcal{H} \mid$ for any $s \in S, x \wedge s \in I\}$ is m-ideal.

Proof. The proof is similar to the proof of Theorem [3.32.

Definition 3.36. Let $\mathcal{H}$ is a hoop. Then $\mathcal{H}$ is called a semi-simple hoop if the intersection of all maximal ideals of $\mathcal{H}$ is $\{0\}$.

Example 3.37. Let $\mathcal{H}$ be the hoop as in Example [.]. Clearly, $\mathcal{M a x}(\mathcal{H})=\{\{0, a\},\{0, b\}\}$ and so $\{0, a\} \cap\{0, b\}=\{0\}$. Hence, $\mathcal{H}$ is semi-simple.

Theorem 3.38. Let $\mathcal{H}$ be a semisimple $\sqcup$-hoop with (DNP). Then $M_{x} \subseteq P_{x}$, for any $x \in \mathcal{H}$.

Proof. Consider $y \in M_{x}$ such that $y \notin P_{x}$. The there exists $P \in \mathcal{M i n}(\mathcal{H})$ such that $x \in P$ and $y \notin P$, and so $(x] \subseteq P$. By Theorem [.]3 we have $(x]^{\perp} \nsubseteq \subseteq P$. Thus there is $a \in(x]^{\perp}$ such that $a \notin P$. So $x \wedge a=0$. Also, from $y, a \notin P$, we get $y \wedge a \notin P$ which implies $y \wedge a \neq 0$. Since $\mathcal{H}$ is semisimple, there is $M \in \mathcal{M a x}(\mathcal{H})$ such $y \wedge a \notin M$, and so $a, y \notin M$. Moreover, from $x \wedge a=0$, we have $x \in M$, which is a contradiction by $y \in M_{x}$. Therefore, $M_{x} \subseteq P_{x}$, for any $x \in \mathcal{H}$.

Corollary 3.39. Let $\mathcal{H}$ be a semisimple $\sqcup$-hoop with (DNP). Then:

(i) If $I$ is a p-ideal, then $I$ is an $m$-ideal of $\mathcal{H}$.

(ii) Every $p$-ideal of $\mathcal{H}$ is $m$-ideal. 


\section{Conclusions and future works}

In this paper, the notion of minimal prime ideals of hoops are defined and investigated some properties of them. Then by using the notion of annihilators, the relation between minimal prime ideals and annihilators is studied. Also, the notion of zero divisors elements of hoops are introduced and proved that the set of all zero divisors of hoops is a union of all minimal prime ideals of hoop. Finally, by using the notions of minimal prime ideals and maximal ideals of hoop, two new ideals as $p$-ideal and $m$-ideal are defined. Then some properties of them are proved and the relation between them is investigated and showed every $p$-ideal of semi-simple hoop is an $m$-ideal of it.

\section{Acknowledgment:}

This research is supported by a grant of National Natural Science Foundation of China (11971384).

\section{References}

[1] M. Aaly Kologani, R.A. Borzooei, On ideal theory of hoop algebras, Mathematica Bohemica, 145(2) (2019), 1-22.

[2] M. Aaly Kologani, Y.B. Jun, X.L. Xin, E.H. Roh, R.A. Borzooei, On co-annihilators in hoops, Journal of Intelligent and Fuzzy Systems, 37(4) (2019), 5471-5485.

[3] M. Aaly Kologani, X.L. Xin, R.A. Borzooei, Y.B. Jun, On annihilators in hoops, submitted.

[4] R.A. Borzooei, M. Aaly Kologani, Local and perfect semihoops, Journal of Intelligent and Fuzzy Systems, 29 (2015), 223-234.

[5] R.A. Borzooei, M. Aaly Kologani, Results on hoops, Journal of Algebraic Hyperstructures and Logical Algebras, 1(1) (2020), 61-77.

[6] B. Bosbach, Komplementäre Halbgruppen. Axiomatik und Arithmetik, Fundamenta Mathematicae, 64 (1969), 257-287.

[7] B. Bosbach, Komplementäre Halbgruppen. Kongruenzen und Quotienten, Fundamenta Mathematicae, 69 (1970), 1-14.

[8] G. Georgescu, L. Leustean, V. Preoteasa, Pseudo-hoops, Journal of Multiple-Valued logic and Soft Computing, 11(1-2) (2005), 153-184.

[9] P. Hájek, Metamathematics of fuzzy logic, Springer Verlag, 4 (1998).

[10] A. Namdar, R.A. Borzooei, Special hoop algebras, Italian Journal of Pure and Applied Mathematics, 39 (2018), 334-349.

[11] A. Namdar, R.A. Borzooei, Nodal filters in hoop algebras, Soft Computing, 22 (2018), 71197128.

[12] A. Namdar, R.A. Borzooei, A. Borumand Saeid, M. Aaly Kologani, Some results in hoop algebras, Journal of Intelligent and Fuzzy Systems, 32 (2017), 1805-1813.

[13] F. Xie, H. Liu, Ideals in pseudo-hoop algebras, Journal of Algebraic Hyperstructures and Logical Algebras, 1(4) (2020), 39-53. 